\title{
Design of stable and broad-band remote vibration controllers for systems with local non-minimum phase dynamics
}

\author{
U. Ubaid, S. Daley, S. A. Pope, and I. Zazas
}

\begin{abstract}
A geometric-based methodology that was recently proposed provides a systematic controller design approach for controlling remote vibration at multiple points using only a restricted number of sensors and actuators. Valuable physical insight into the existence of control solutions for vibration attenuation at multiple locations is retained with this approach in contrast to alternatives, such as $\mathcal{H}_{2}$ and $\mathcal{H}_{\infty}$ methods. A drawback of the existing geometric design approach is that the controller implementation for the broad band case incorporates an inverted local control path transfer function. When the sensor and actuator are non-collocated or when there is significant latency or phase lag in the system, the local control path model will have non-minimum phase characteristics. Therefore the resulting controller for this situation will itself be unstable due to the inclusion of an inverted non-minimum phase transfer function. In this paper a systematic procedure is presented that extends the previous work and which yields both a stable and stabilising controller without requiring a minimum phase control path assumption. Furthermore, robustness against control spillover at out of band frequencies is incorporated within this modified design procedure without deteriorating controller performance within the design bandwidth. The detailed control design procedure is illustrated using a simulated beam vibration problem. Finally, the design approach is experimentally validated using a test rig that replicates the problem of vibration transmission in rotary propulsion systems.
\end{abstract}

\section{INTRODUCTION}

$\mathbf{T}$ HE REQUIREMENT to reduce vibration at specific points on a flexible structure can be achieved using active control systems in a feedback configuration whereby sensors and actuators are located at these same points in a collocated or non-collocated set-up [1], [2]. However, for complex interconnected structures it is not always feasible to locate sensors and actuators at the points where vibration attenuation is desired. This restriction arises from practical constraints and can be due to cost, space or environmental considerations. In such circumstances, feedback control systems have to be employed at a restricted number of easily accessible locations and therefore the performance of the closed loop system cannot simply be assessed in terms of the measurement obtained at the local error sensors.

A typical example of such a problem is the vibration encountered due to aerodynamic loading on helicopter blades.

U. Ubaid, S. Daley and I. Zazas are with the Institute of Sound and Vibration Research, University of Southampton, Southampton, SO17 1BJ, UK, e-mail: (u.ubaid, s.daley, i.zazas)@soton.ac.uk.

SA. Pope is with the Department of Automatic Control and Systems Engineering, University of Sheffield, Sheffield, S1 3JD, UK, e-mail: s.a.pope@sheffield.ac.uk
The unsteady forces experienced by the rotor blades are generally transmitted at multiples of the blade passing frequency through the hub to the fuselage. Although some active damping approaches that are applied directly to the rotating blades have been proposed [3], [4] it is more practical to apply control across the gearbox at the opposite end of the propeller shaft [5]. Although the latter approach will reduce the locally measured fuselage vibration, the change in impedance seen by the shaft can lead to an increase in blade vibration. This effect has been demonstrated within the context of maritime vehicles in [6], [7].

A systematic controller design approach was developed previously that addresses such problems and also provides insight into the conflicting requirements of vibration attenuation at local and remote points ${ }^{1}$ on a structure [7]. The approach enables global optimisation for discrete frequencies with a single loop collocated control architecture. The controller is defined as a function of a single frequency-dependent design freedom parameter. The feasible solutions of simultaneous vibration reduction at both the local and remote points can be deduced using this single design parameter. An advantage of the method is that the feasible set of attenuation solutions are presented in a simple graphical form and the designer can select the controller to satisfy a variety of frequency dependent control objectives. As a result the approach is closely related to classical loop shaping procedures. For the task of attenuation over an arbitrary frequency band, the controller implementation proposed in [8] incorporates an inverted local control path transfer function. As a result an assumption of an ideally collocated sensor and actuator pair has to be made in order to ensure that the control path dynamics are minimum phase and consequently that the controller is itself stable. However in practice even for the collocated case the experimentally derived control path transfer function can be non-minimum phase since it incorporates sensor, actuator and signal conditioning dynamics together with acquisition latencies. For the non-minimum phase case the controller given by the method in [8] would itself be an unstable function by virtue of the right half plane zeros present in the control path transfer function. Even if the closed loop system is internally stable, the implementation of an unstable controller is not practical. As the closed loop system is only conditionally stable, any scenario that leads to a variation in gain would

\footnotetext{
${ }^{1}$ The term local point is used to refer to the location of the sensor and actuator on the structure while a remote point refers to any other location where a disturbance force acts or where vibration reduction is desired.
} 
lead to instabilities [9]. This can occur for example during commissioning and start-up as the controller gain is gradually increased to the nominal and so may pass through regions of instability. One of the solutions to address implementation of unstable controllers involves switching between the Youla parameterisation of a stable controller and the unstable controller until the latter is fully active [10]. However, this adds complexity to the implementation and it is more desirable to have a controller function that is strongly stabilising.

Moreover, the unmeasured out of band dynamics at high frequencies can lead to control spillover with the potential to cause instability. The gain of the open loop system must roll-off above the disturbance attenuation bandwidth, which for most practical vibration control applications will be in the low-mid frequency region. This satisfies noise rejection requirements and provides robustness to unmodelled dynamics. Also, in certain applications it is desired to have a low control gain at low frequencies. This can, for example, be needed to avoid stroke/force saturation due to resonance in proof mass actuators [11], [12], or to avoid problems associated with low frequency accelerometer inaccuracy. In order to address these problems, a modified design approach is proposed in this paper for the general non-minimum phase case which gives a strongly stabilising controller that is also robust to unmodelled dynamics.

The paper is structured as follows: In Section II a control design procedure for a robust stable and stabilising controller is presented. The efficacy of the design approach is illustrated with simulation results using a beam model in Section III. In Section IV the control design is validated experimentally using a rig that replicates the vibration problems associated with propeller blade excitation that are encountered in aerospace and maritime propulsion systems. The experimental results are followed by concluding remarks in Section V.

\section{STABLE CONTROLLER FOR NON-MINIMUM PHASE DYNAMICS}

The original geometric control design procedure was developed (see Section 2 of [8]) for a system described by the following frequency response function (FRF).

$$
\left[\begin{array}{l}
y(\mathrm{j} \omega) \\
z(\mathrm{j} \omega)
\end{array}\right]=\left[\begin{array}{ll}
g_{11}(\mathrm{j} \omega) & g_{12}(\mathrm{j} \omega) \\
g_{21}(\mathrm{j} \omega) & g_{22}(\mathrm{j} \omega)
\end{array}\right]\left[\begin{array}{l}
u(\mathrm{j} \omega) \\
d(\mathrm{j} \omega)
\end{array}\right]
$$

where $y(j \omega), z(j \omega), u(j \omega)$ and $d(j \omega)$ represent the locally measured response, the remote vibration response, the control input and the disturbance input, respectively. Although the motivation behind this control design is the unavailability of sensors or actuators at the remote location during operation, it is assumed that the remote response is available for measurement during a commissioning stage. As noted in the introduction this may be due to operational cost constraints or in the case of rotary propulsion, environmental considerations. The local response is used as a feedback signal to generate the control input according to

$$
u(\mathrm{j} \omega)=-k(\mathrm{j} \omega) y(\mathrm{j} \omega)
$$

The objective is to obtain a controller $(k(\mathrm{j} \omega))$ that reduces both the local $(y(\mathrm{j} \omega))$ and remote $(z(\mathrm{j} \omega))$ vibration response over a target frequency span. The local control path transfer function $g_{11}(s)$ was assumed to be minimum phase in the original formulation [8]. The control design for the general case of nonminimum phase control path dynamics is addressed here. Also, the design procedure addresses the requirement to suppress control spill-over at out of band frequencies. According to this method, the modified design freedom parameter, $\gamma$, is related to the sensitivity function $S(\mathrm{j} \omega)$ and complementary sensitivity function $T(\mathrm{j} \omega)$, of the local feedback loop, as

$$
\gamma(\mathrm{j} \omega)=\frac{[S(\mathrm{j} \omega)-1]}{\mathcal{B}_{11}(\mathrm{j} \omega) W_{\gamma}(\mathrm{j} \omega)}=\frac{-T(\mathrm{j} \omega)}{\mathcal{B}_{11}(\mathrm{j} \omega) W_{\gamma}(\mathrm{j} \omega)}
$$

where $\mathcal{B}_{11}(j \omega)$ is the frequency response of an all pass transfer function formed from the decomposition of the nonminimum phase transfer function $g_{11}(s)$ into a minimum phase counterpart $g_{\mathrm{MP}}(s)$ as $g_{11}(s)=g_{\mathrm{MP}}(s) \mathcal{B}_{11}(s)$, and $W_{\gamma}(\mathrm{j} \omega)$ is the frequency response of a weighting filter chosen to suppress control spillover. Using (1), (2) and (3) the closed loop local and remote vibration responses can be denoted in terms of this design freedom variable as (4) and (6) respectively

$$
y(\mathrm{j} \omega)=\left[1+\frac{\gamma(\mathrm{j} \omega)}{U_{\mathrm{L}}(\mathrm{j} \omega)}\right] g_{12}(\mathrm{j} \omega) d(\mathrm{j} \omega)
$$

where

$$
\begin{gathered}
U_{\mathrm{L}}(\mathrm{j} \omega)=\frac{1}{\mathcal{B}_{11}(\mathrm{j} \omega) W_{\gamma}(\mathrm{j} \omega)} \\
z(\mathrm{j} \omega)=\left[1+\frac{\gamma(\mathrm{j} \omega)}{U_{\mathrm{R}}(\mathrm{j} \omega)}\right] g_{22}(\mathrm{j} \omega) d(\mathrm{j} \omega)
\end{gathered}
$$

where

$$
U_{\mathrm{R}}(\mathrm{j} \omega)=\frac{g_{11}(\mathrm{j} \omega) g_{22}(\mathrm{j} \omega)}{\mathcal{B}_{11}(\mathrm{j} \omega) W_{\gamma}(\mathrm{j} \omega) g_{12}(\mathrm{j} \omega) g_{21}(\mathrm{j} \omega)}
$$

Comparing the closed loop responses given by (4) and (6) with the open loop response, it can be seen that simultaneous reduction in both outputs is possible using a controller that minimises the terms inside the brackets in (4) and (6). This minimisation can be stated for a discrete frequency $\omega_{0}$, in a similar manner to the original formulation described in [7], as (8) and (9) respectively.

$$
\begin{aligned}
& \left|\gamma\left(j \omega_{0}\right)+U_{\mathrm{L}}\left(\mathrm{j} \omega_{0}\right)\right|<\left|U_{\mathrm{L}}\left(\mathrm{j} \omega_{0}\right)\right| \\
& \left|\gamma\left(\mathrm{j} \omega_{0}\right)+U_{\mathrm{R}}\left(\mathrm{j} \omega_{0}\right)\right|<\left|U_{\mathrm{R}}\left(\mathrm{j} \omega_{0}\right)\right|
\end{aligned}
$$

Inequality (8) is an algebraic constraint which describes a region in the $\gamma$-plane inside a unit radius circle, i.e. $\left|U_{\mathrm{L}}\left(\mathrm{j} \omega_{0}\right)\right|=1$, centred on $-U_{\mathrm{L}}\left(\mathrm{j} \omega_{0}\right)$. Similarly, inequality (9) describes the region inside a circle with centre $-U_{R}\left(j \omega_{0}\right)$ and radius $\left|U_{\mathrm{R}}\left(\mathrm{j} \omega_{0}\right)\right|$ in the $\gamma-$ plane. Simultaneous attenuation of $y\left(j \omega_{0}\right)$ and $z\left(j \omega_{0}\right)$ is possible, therefore, if both circles intersect in the $\gamma$-plane. It can be seen, however, that if $\left|g_{12}\left(\mathrm{j} \omega_{0}\right) g_{21}\left(\mathrm{j} \omega_{0}\right)\right|<<\left|g_{11}\left(\mathrm{j} \omega_{0}\right) g_{22}\left(\mathrm{j} \omega_{0}\right)\right|$, then the circle for reduction of the remote response has a very large radius with its centre $-U_{\mathrm{R}}\left(j \omega_{0}\right)$ far from the centre of the local vibration reduction circle $-U_{\mathrm{L}}\left(\mathrm{j} \omega_{0}\right)$. The control authority for the remote response will be low in this case. This can happen, 
for example, if the control input or the remote output is close to a node of a resonant mode that is to be controlled.

Specific values for the design freedom parameter are selected at discrete frequencies from within the region of intersection of the circles depending upon the design objectives. In a similar manner to the procedure proposed in [8] these optimal values for the design freedom parameter within the design band $\left[\omega_{L}, \omega_{H}\right]$ can be interpolated by a stable transfer function $\gamma(s)$, using an algorithm of the Nevanlinna-Pick interpolation problem [13]. Using this technique a set of complex $\gamma$ values $\gamma_{1}, \ldots, \gamma_{N}$ selected at $N$ discrete frequency points $\omega_{1}, \ldots, \omega_{N}$ can be interpolated to obtain a continuoustime transfer function $\gamma(s)$ such that $\gamma\left(\mathrm{j} \omega_{i}\right)=\gamma_{i}$ for all $i=1, \ldots, N$. The controller is then implemented as

$$
k(s)=-\frac{\gamma(s) W_{\gamma}(s)}{\left[1+\gamma(s) \mathcal{B}_{11}(s) W_{\gamma}(s)\right] g_{\mathrm{MP}}(s)}
$$

The number of excess poles of the controller is determined by the difference between the number of excess poles of $W_{\gamma}(s)$ and the number of excess poles of $g_{\mathrm{MP}}(s)$. Hence, even if $\gamma(s)$ is not a strictly proper transfer function, the final controller can be made to be strictly proper and hence causal without performance loss by selection of a suitable weighting function $W_{\gamma}(s)$. In addition, since $g_{\mathrm{MP}}(s)$ has no RHP zero(s), a controller given by (10) is itself stable if $\gamma(s)$ is a stable transfer function and the Nyquist contour of $\gamma(\mathrm{j} \omega) \mathcal{B}_{11}(\mathrm{j} \omega) W_{\gamma}(\mathrm{j} \omega)$ does not enclose the $(-1,0)$ point. This follows from the form of the controller structure given in (10) since this is equivalent to a closed loop feedback system having an open-loop frequency response function of $\gamma(\mathrm{j} \omega) \mathcal{B}_{11}(\mathrm{j} \omega) W_{\gamma}(\mathrm{j} \omega)$. The main steps in the controller design process are outlined in Fig. 1.

\section{Illustrative Design EXAMPle}

\section{A. Beam model}

In this section the response of a slender beam, assuming Euler Bernoulli beam theory for a simply supported boundary condition, is used to illustrate the strongly stabilising remote vibration control design technique developed in the paper and compare the performance with an $\mathcal{H}_{\infty}$ controller design. A $1 \mathrm{~m}$ long steel beam with density $\rho=7850 \mathrm{~kg} / \mathrm{m}^{3}$ and Young's modulus $E=21 \times 10^{10} \mathrm{~N} / \mathrm{m}^{2}$ is considered. It has a crosssection of $2.0 \times 2.0 \mathrm{~cm}$. A schematic drawing showing the location of control input, disturbance excitation, local and remote outputs is shown in Fig. 2. $\alpha \in[0,1]$ represents a gain that is gradually increased to the nominal during controller commissioning. To develop the model, vertical translational displacement and angular rotation degrees of freedom are considered for 10 equal beam segments which gives an approximation for the first 20 modes of the beam. The control input $(u)$ acts as a point translation force at a distance $0.5 \mathrm{~m}$ while the feedback signal $(y)$ is the displacement measured at a distance $0.1 \mathrm{~m}$, from one end of the beam. Although sensor and actuator dynamics are not included in the model the non-collocated arrangement imparts non-minimum phase characteristics on the local control path transfer function $g_{11}(s)$ and so serves to illustrate the key aspects of the design

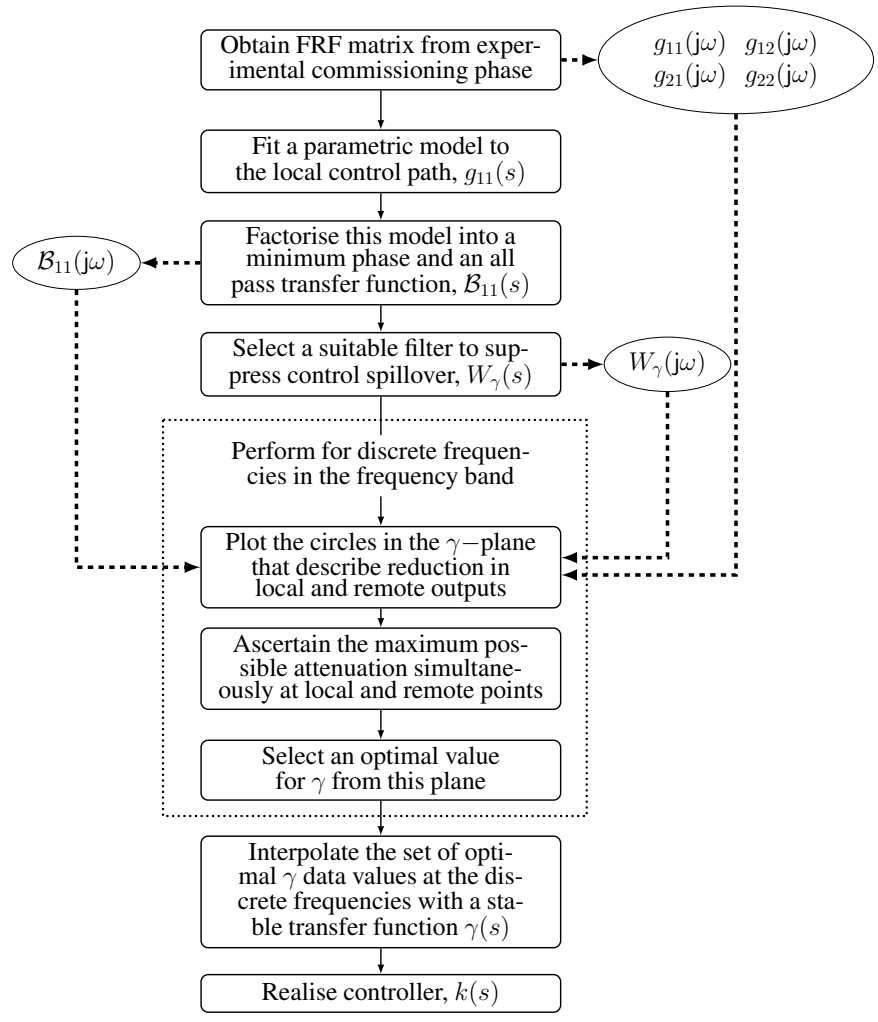

Fig. 1. Flow chart showing the main steps of the geometric based control design process

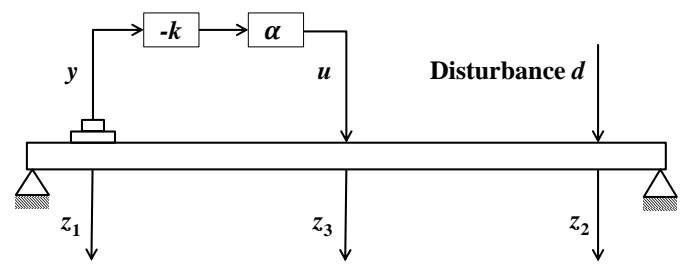

Fig. 2. A schematic representation of the local and remote control set-up for the beam simulation example.

process. The primary excitation $(d)$ acts as a point force at a distance of $0.9 \mathrm{~m}$ from the same end of the beam. In addition to the local output, the response at two remote points at distances $0.9 \mathrm{~m}$ and $0.5 \mathrm{~m}$ are also considered. The design goal addressed is the development of a feedback controller that achieves simultaneous attenuation at the local and remote points for the first four resonant modes.

\section{B. $\mathcal{H}_{\infty}$ controller design for active damping}

To provide a benchmark for the approach proposed in this paper the feasibility of reducing the resonant peaks in the magnitude of response at the local and remote points is initially investigated for different $\mathcal{H}_{\infty}$ controller designs. The conflicting requirements for remote and local vibration reduction is illustrated using different error signals to be minimised for $\mathcal{H}_{\infty}$ controller design. Initially, an $\mathcal{H}_{\infty}$ controller is synthesised using MATLAB's hinfsyn function (which is based on the method described in [14]), in order to minimise only the local output $\left(z_{1}\right)$. Using the resulting controller the magnitude of the 
closed loop response at the local point, Fig. 3, shows reduction of the lower frequency resonant modes. However, the closed loop response of the remote point $\left(z_{2}\right)$ at $0.9 \mathrm{~m}$ in Fig. 4 shows that the second and fourth resonant peaks are not minimised. Moreover, there are enhancements at the frequencies of these two resonances in the closed loop output of the midpoint $\left(z_{3}\right)$ as seen in Fig. 5. If the error signal to be minimised is chosen as only the remote output $\left(z_{2}\right)$ at $0.9 \mathrm{~m}$ then as can be seen from Fig. 4 the $\mathcal{H}_{\infty}$ controller attenuates all the low frequency resonant modes in this response. However, the second and fourth resonant modes are not minimised in the output at the local point $\left(z_{1}\right)$ as seen in Fig. 3. Also, the controller causes enhancement at these two frequencies in the closed loop output at $0.5 \mathrm{~m}\left(z_{3}\right)$ as seen in Fig. 5. Selecting the error signal to be minimised as both the local and remote output gives a closed loop response that is similar to one of the above designs depending upon the weighting function used. It is clear, therefore, that it is not possible to synthesise an $\mathcal{H}_{\infty}$ controller that can minimise the response in both $z_{1}$ and $z_{2}$ at the second and fourth resonant peaks simultaneously. However, the tradeoff between these two conflicting performance requirements is not evident to the control designer. The additional insight that can be obtained using the geometric design approach at the frequencies of the second and fourth bending modes is illustrated in the next section. It should also be noted that the two $\mathcal{H}_{\infty}$ controllers synthesised in this section are themselves unstable and therefore difficult to implement in practice. This is verified for the first controller in Fig. 6(a) which shows an unstable response when the controller commissiong gain is $1 / 10^{t h}$ of the nominal value (i.e. $\alpha=0.1$ ). The closed loop system for the second $\mathcal{H}_{\infty}$ controller is similarly unstable for $\alpha=0.1$.

\section{Trade-off in performance using $\gamma$-plane}

The first step in the geometric portrayal of feasible vibration attenuation is the computation of the centre of the circles corresponding to reduction at the local and remote points for discrete frequencies using inequalities (8) and (9). $\mathcal{B}_{11}(s)$ includes seven real right half plane zeros. The filter $W_{\gamma}(s)$ is chosen here as a fourth order band-pass butterworth filter with cut-on and cut-off frequencies of $22 \mathrm{~Hz}$ and $1.5 \mathrm{kHz}$, respectively. Circles in the $\gamma$-plane corresponding to closed loop vibration levels at the local point (at $0.1 \mathrm{~m}$ ) and two remote points (at $0.5 \mathrm{~m}$ and $0.9 \mathrm{~m}$ ) are plotted for the first four resonant frequencies in Fig. 7. It can be seen that for the first and third bending modes the circles nearest to the resonant frequencies (at $47 \mathrm{~Hz}$ and $422 \mathrm{~Hz}$ ) for both the remote points and the local point almost completely overlap. As a result, in these frequency regions very high levels of global reduction are feasible as the controller can be realised using values of $\gamma$ that are close to the centres of all circles. However, close to the second and fourth bending mode frequencies at $188 \mathrm{~Hz}$ and $752 \mathrm{~Hz}$, the circles for reduction at $0.9 \mathrm{~m}$ lie diametrically opposite to the local vibration reduction circles. Therefore, from the plot of circles in the $\gamma$-plane it can be seen that simultaneous reduction in the output at $0.1 \mathrm{~m}$ and $0.9 \mathrm{~m}$ is not possible for the second and fourth resonant frequencies.

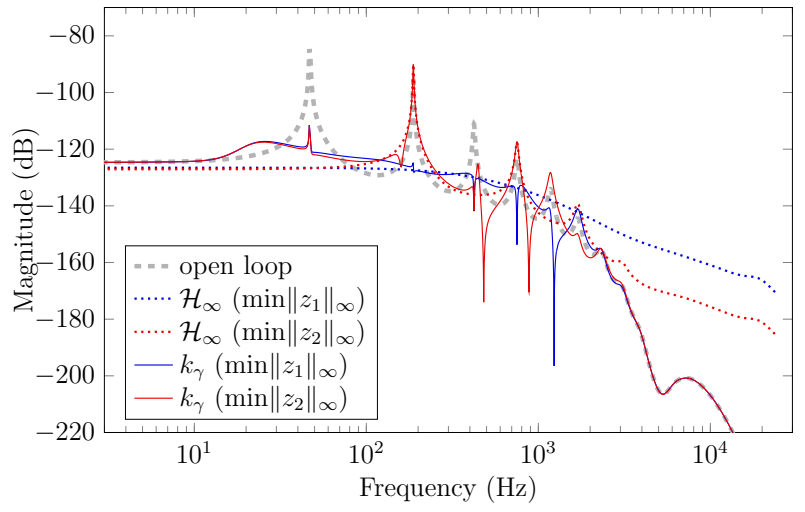

Fig. 3. Magnitude of the frequency response at local point $(0.1 \mathrm{~m})$ for an input at remote point $(0.9 \mathrm{~m})$ on the beam without feedback control (dashed), with $\mathcal{H}_{\infty}$ feedback controller (dotted) and geometric design controller (solid), for error signal minimisation of the local output (blue) and remote output (red).

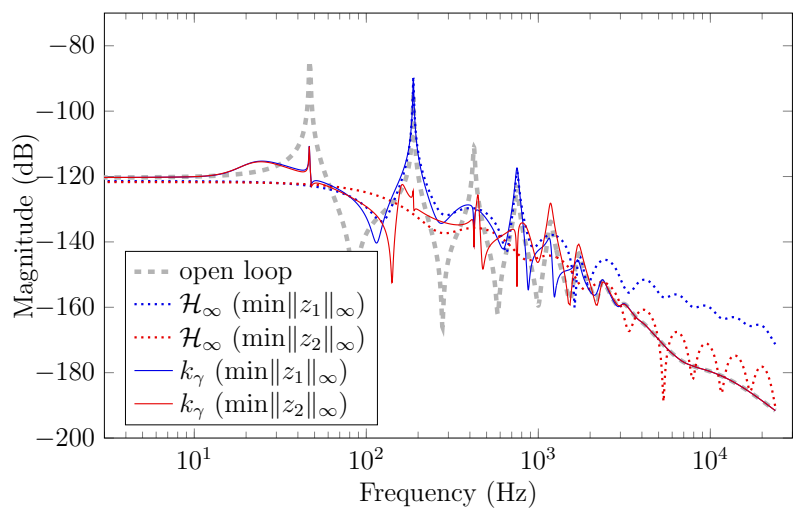

Fig. 4. Magnitude of the frequency response at remote location $(0.9 \mathrm{~m})$ for an input at this same location without feedback control (dashed), with $\mathcal{H}_{\infty}$ feedback controller (dotted) and geometric design controller (solid), for error signal minimisation of the local output (blue) and remote output (red).

Also, a consequence of the midpoint of the beam being a node of the second and fourth mode is that the circles for reduction at $0.5 \mathrm{~m}$ are smaller and lie close to the origin. As a result reductions in levels at the local point or remote point at $0.9 \mathrm{~m}$ around these frequencies would necessitate an increase in levels at the midpoint.

\section{Results of geometric controller}

Geometric controllers are designed for the two cases of optimal reduction at the local $(0.2 \mathrm{~m})$ or remote point $(0.9 \mathrm{~m})$, as in the case of $\mathcal{H}_{\infty}$ controller design. Target values for the design parameter are selected at discrete frequencies from near the centre of the circles denoting local vibration reduction (blue circles in Fig. 7) in the first case and for remote vibration reduction (red circles in Fig. 7) in the second case. The magnitude of the closed loop frequency responses at the local $(0.1 \mathrm{~m})$ and remote points $(0.9 \mathrm{~m}$ and $0.5 \mathrm{~m})$ are shown in Figs. 3, 4 and 5 for the two cases of controller design. It can be seen that the first four resonant peaks in the local output are attenuated using a controller that is realised using $\gamma$ values selected for attenuation at only this point. As predicted by the results shown in Fig. 7 this controller 


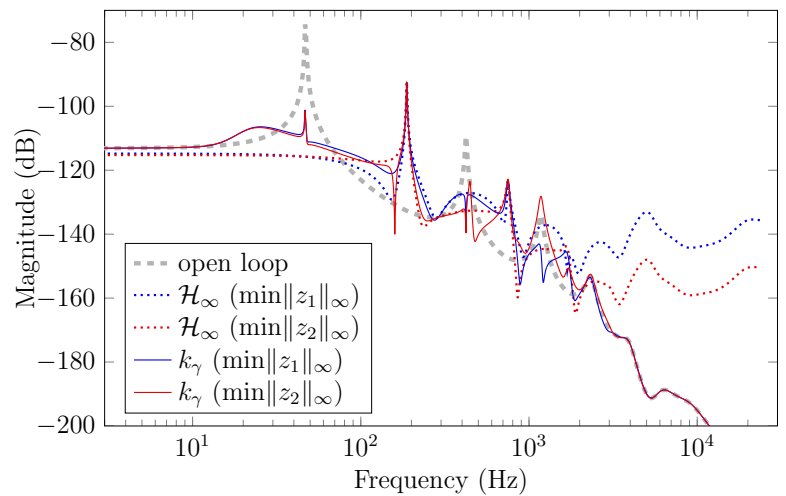

Fig. 5. Magnitude of the frequency response at mid point of beam $(0.5 \mathrm{~m})$ for an input at remote point $(0.9 \mathrm{~m})$ without feedback control (dashed), with $\mathcal{H}_{\infty}$ feedback controller (dotted) and geometric design controller (solid), for error signal minimisation of the local output (blue) and remote output (red).

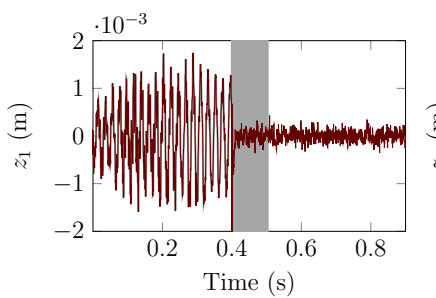

(a)

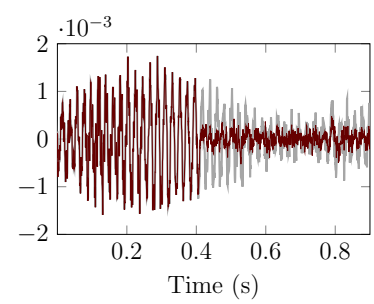

(b)
Fig. 6. Time domain response (a) $z_{1}(t)$ with $\mathcal{H}_{\infty}$ controller, designed for minimisation of $\left\|z_{1}\right\|_{\infty}$, switched $\mathrm{ON}$ at $t=0.4 \mathrm{~s}$ and (b) $z_{1}(t)$ with geometric controller, designed for minimisation of $\left\|z_{1}\right\|_{\infty}$, switched ON at $t=0.4 s$, for $\alpha=0.1(\longrightarrow)$ and $\alpha=1(-)$

leads to enhancement at the midpoint and no attenuation in the output at the remote point, for the second and fourth resonant frequencies. The second controller which is designed for optimum attenuation of output at the remote point $(0.9 \mathrm{~m})$ attenuates the low frequency resonant modes. However, this also causes enhancement in the response at the midpoint while giving no attenuation of the second and fourth resonances at the local point. The closed loop performance obtained using these two controllers are broadly similar to that obtained using the $\mathcal{H}_{\infty}$ controllers but with an additional gain roll-off at higher frequencies due to the filter $W_{\gamma}(s)$. However unlike the $\mathcal{H}_{\infty}$ controllers both of these controllers are stable functions thereby enabling a straightforward implementation. This can be verified from the simulation of the closed loop in Fig. 6(b) that shows the response for gains lower than the nominal. Moreover, the methodology proposed here enables the feasible performance to be easily assessed using a simple graphical portrayal of the design freedom (Fig. 7).

\section{EXPERIMENTAL VALIDATION}

\section{A. Blade test rig}

The efficacy of the control design methodology is further demonstrated in this section using the experimental test rig that was previously used to illustrate the geometric-based controller designed for tonal excitation [7]. This facility was constructed to replicate the problems associated with vibration transmission in rotary based propulsion systems that were discussed in the introduction. As illustrated in Fig. 8 unsteady forces generated at the blade propagate along the shaft and transmit via the thrust bearing into the supporting structure. A strategy for remote vibration attenuation is to control both the blade vibration and onward transmission through the propeller shaft using only sensors and actuators located at the thrust bearing end of the shaft ${ }^{2}$. Although this represents a case where it is not practically viable to provide in-service sensing and actuation of the blade, it is assumed that all the necessary transfer functions can be identified from measurements during a commissioning phase when the shaft is stationary.

The test rig is made up of a long hollow shaft pinned at one end to the centre of a flexible beam element which represents the rotor blade. The other end of the shaft is fixed to a steel block rigidly connected to the supporting foundation, which represents the thrust bearing. The beam can be excited by two small inertial shakers to simulate the effect of hydrodynamic or aerodynamic loading on the blades (represented by $d$ in Fig. 8); and a shaker at the fixed end of the shaft implements the control force $(u)$. An accelerometer measures the acceleration of the block which is used as a feedback signal $(y)$. The vibration in the beam is measured by two accelerometers located close to the shakers for solely monitoring the performance. The frequency response of the summed beam acceleration $(z)$ to a common excitation signal for the shakers located on the beam shows that the first bending mode resonance occurs around $244 \mathrm{~Hz}$ (Fig. 10). This resonance leads to a peak in the transmission along the shaft, as can be seen in Fig. 9 for the thrust block response to the same excitation signal. It should be noted that unlike the beam simulation example in the previous section, the control force is applied here at a non-resonating part of the interconnected structure.

\section{B. $\mathcal{H}_{\infty}$ controller}

In order to highlight the potential benefits of the design approach proposed in this paper an $\mathcal{H}_{\infty}$ controller is considered for the feedback path at the thrust block. The design specifications are selected so as to minimise the response of the thrust block. The controller design requires parametric model identification for all four paths between the control and disturbance inputs, and the local and remote outputs. The closed loop response predictions using this controller are shown in Figs. 9 and 10 . The $\mathcal{H}_{\infty}$ controller gives a reduction of around $10 \mathrm{~dB}$ in the thrust block response at $244 \mathrm{~Hz}$, however, there is deterioration at low frequencies due to the unmodelled actuator dynamics. The closed loop blade response is similar to the open loop case. The controller has four unstable poles at $12.89 \pm \mathrm{j} 1526.58$ and $0.84 \pm \mathrm{j} 5171.54$. Therefore its implementation is not straightforward and as a result experimental validation is not undertaken.

\section{Geometric control design}

Unlike $\mathcal{H}_{\infty}$ controller design the geometric-based control design requires parametric model identification for only the

\footnotetext{
${ }^{2}$ Note that this general concept is the subject of several patents
} 


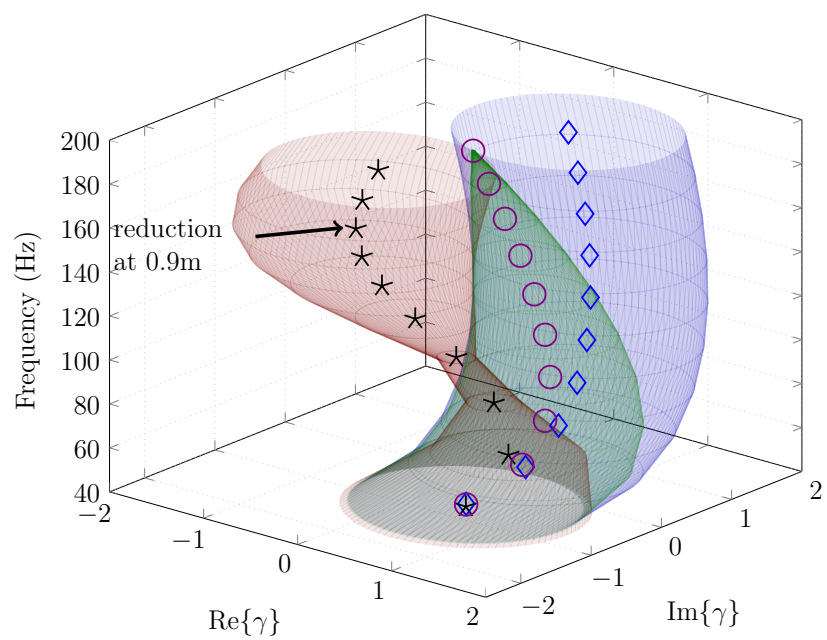

(a)

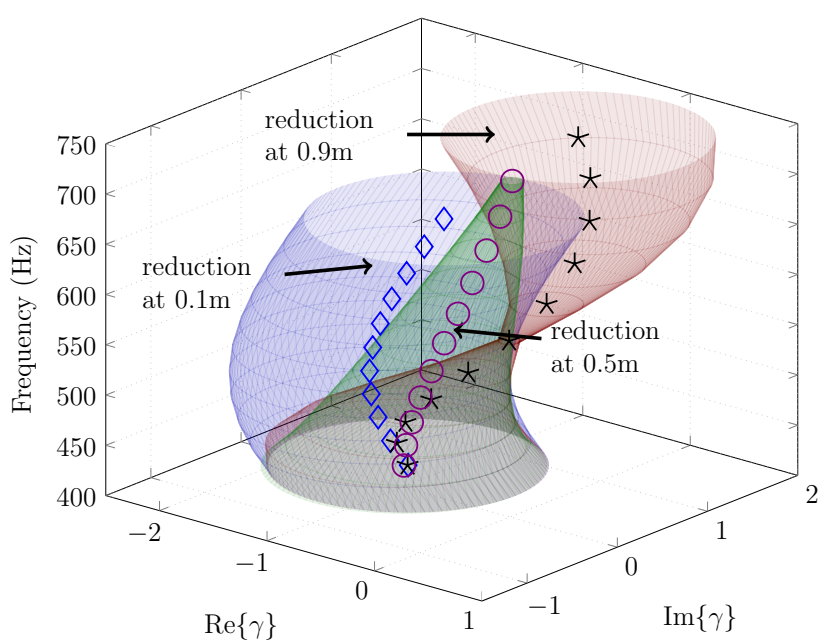

(b)

Fig. 7. Portrayal of vibration attenuation as regions enclosed by circles corresponding to points at distances $0.1 \mathrm{~m}$ (blue), $0.9 \mathrm{~m}$ (red) and $0.5 \mathrm{~m}$ (green) from one end of the beam in $\gamma$-plane and their centres $(\diamond, \star$ and $\bigcirc$, respectively) which denote maximum attenuation in the respective outputs for frequencies around (a) first and second bending mode and (b) third and fourth bending mode.

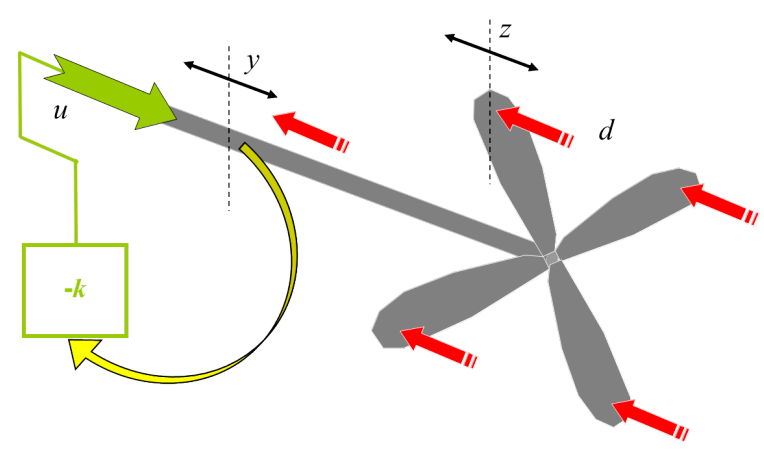

Fig. 8. Disturbance excitation is the aerodynamic or hydrodynamic loading on the rotor blades. At the resonance of the blade elements there is a peak in the propagation of disturbance along the shaft which is to be attenuated by a correcting force applied at the non-resonating part of the structure

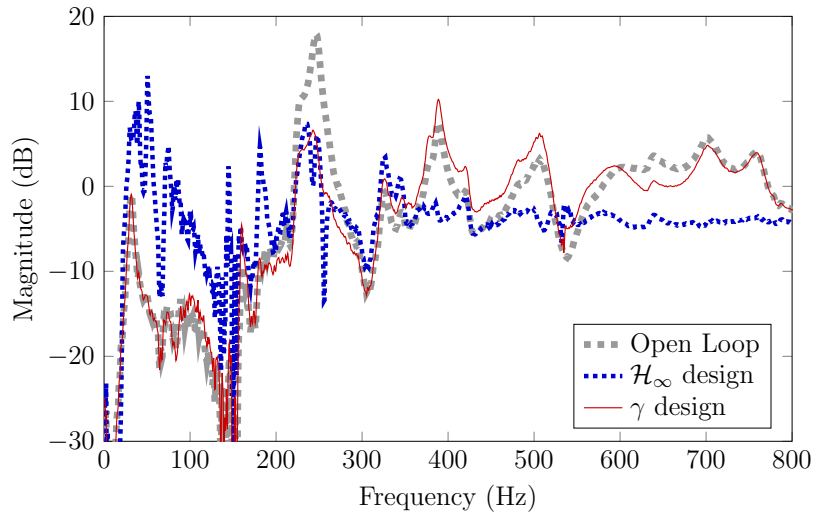

Fig. 9. Predicted magnitude of the frequency response of the thrust block output to the blade disturbance input without feedback control (dashed), $\mathcal{H}_{\infty}$ control (dotted) and geometric based control (solid)

local control path transfer function. The first step in the control design is to determine a linear time invariant model for the

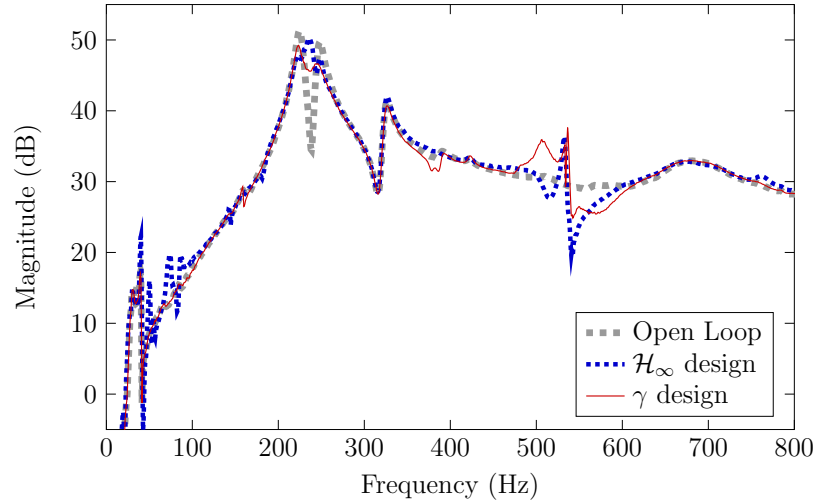

Fig. 10. Predicted magnitude of the frequency response of the summed blade vibration output to the blade disturbance input without feedback control (dashed), $\mathcal{H}_{\infty}$ control (dotted) and geometric based control (solid)
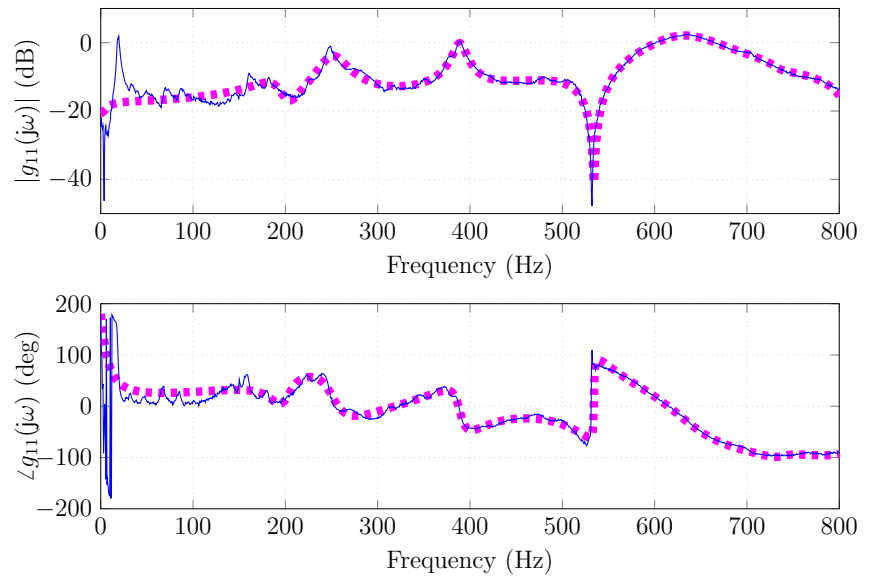

Fig. 11. Magnitude and phase of the frequency response of the thrust block acceleration to control input, measured (solid) and a model (dashed) 


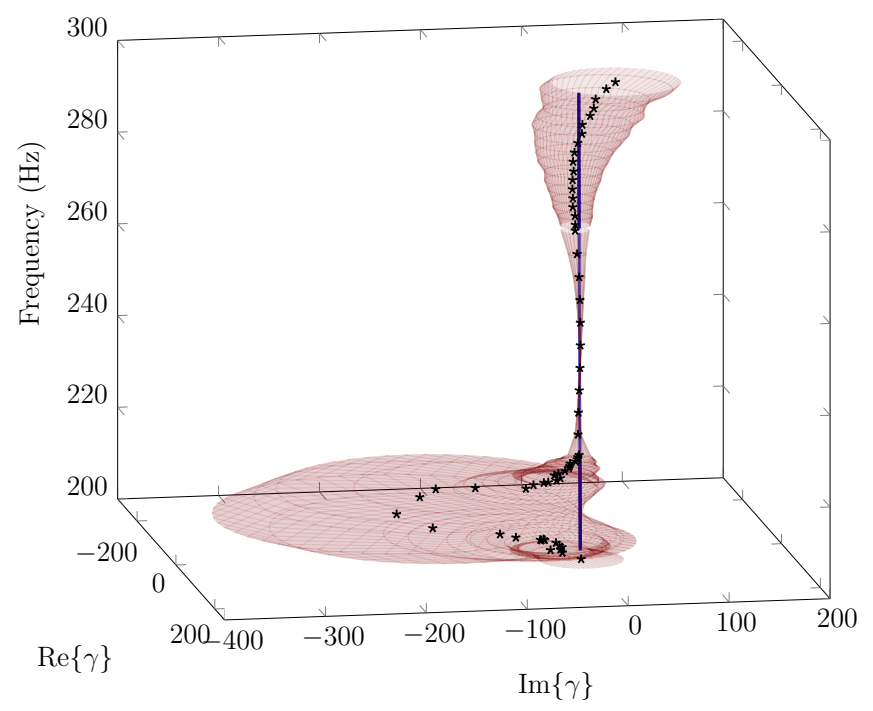

Fig. 12. The attenuation in blade vibration for discrete frequencies from 200 $\mathrm{Hz}$ to $300 \mathrm{~Hz}$ can be achieved using values for $\gamma$ from inside the red region and maximum attenuation can be achieved when it is selected from the centre shown as $\star$. Unit radius circles corresponding to attenuation in thrust block vibration appear as a line passing through the origin

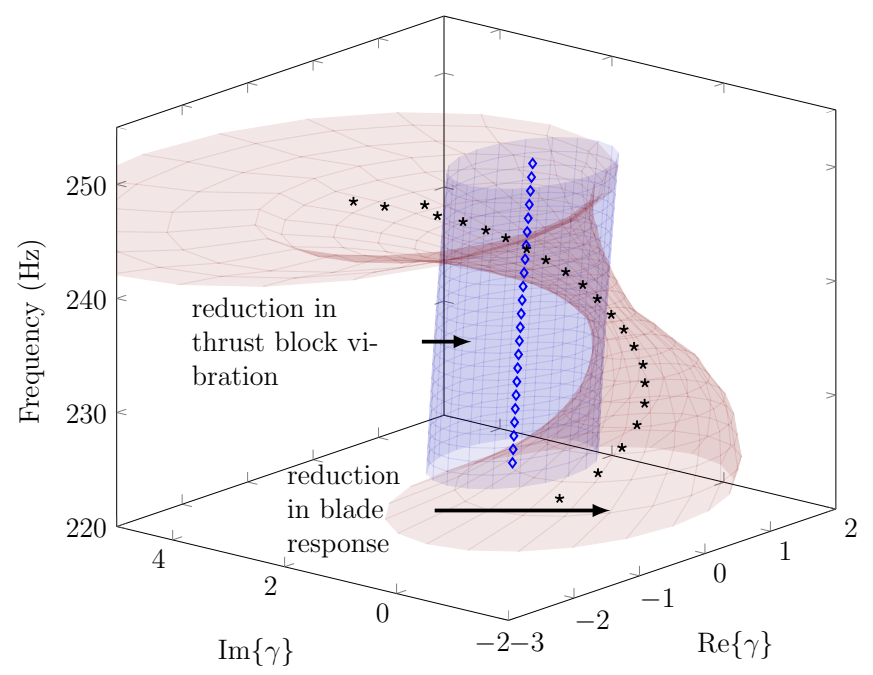

Fig. 13. Circles as shown in Fig. 12 zoomed into frequency range $220 \mathrm{~Hz}$ to $250 \mathrm{~Hz}$

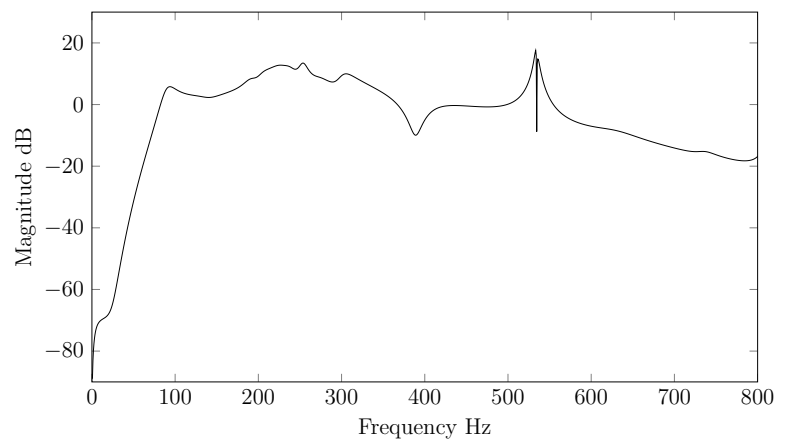

Fig. 14. Magnitude of the frequency response of the geometric controller open loop control path between the control shaker and thrust block acceleration. A 15th order transfer function model using the MATLAB's built-in function invfreqs (based on the method defined in [15]) is identified for the open loop control path as shown in Fig. 11, which is non-minimum phase with one right half plane zero. It will be noted that the model does not include the low frequency resonant modes associated with the actuator dynamics since this is outside the frequency band of interest. The filter $W_{\gamma}(s)$ is therefore chosen as an 8th order bandpass butterworth filter with low cut-on and high cut-off frequencies as $100 \mathrm{~Hz}$ and $600 \mathrm{~Hz}$ respectively. The circles in the $\gamma$-plane corresponding to reduction in vibration levels at the local and remote points for frequencies around the first bending mode are shown in Fig. 12. The circles corresponding to a reduction in the blade vibration are very large from $200 \mathrm{~Hz}$ to $220 \mathrm{~Hz}$ and above $270 \mathrm{~Hz}$. The distance between the centre of both circles will be large and so it will not be possible to achieve considerable vibration reduction at the blade end without amplifying vibration levels at the thrust bearing end of the shaft. In practice however the controller objective is to minimise the transmission through the thrust bearing subject to the constraint that the blade vibration is not enhanced. It can be seen that $\gamma$ points that achieve this objective can be obtained by selecting points that are close to the centre of the unit circle but that remain within the red circles. However as shown in Fig. 13 the $\gamma$ points for frequencies from $220 \mathrm{~Hz}$ to $245 \mathrm{~Hz}$ selected from within the region of intersection of both sets of circles would lie close to the origin. This would correspond to low control action and hence the intended attenuation of thrust bearing response would not be achieved. Therefore the $\gamma$ points are selected from near the centre of the blue circles which will lead to a slight deterioration in blade response near this frequency. A stable $\gamma(s)$, determined using the Nevanlinna-Pick interpolation technique, also satisfies the controller stability condition. However, due to inversion of the minimum phase counterpart of the control path transfer function the antiresonances of this transfer function can manifest as peaks in the controller FRF. Here, a minimum in the control path FRF as seen in Fig. 11 associated with a lightly damped conjugate pair of zeros could cause enhancement in the closed loop response or even reduce stability margins. This could either be dealt with by excluding this region from the parametric model or by further shaping the design freedom parameter. However, a notch filter is utilised here to reduce the peak at this frequency without affecting the gain or phase of the controller below $500 \mathrm{~Hz}$. The magnitude of the controller frequency response is shown in Fig. 14. The predicted magnitude of the closed loop frequency response of the thrust block and blade vibration using this controller are plotted in Figs. 9 and 10. They show good attenuation of the peak response in the thrust block at $244 \mathrm{~Hz}$, while slightly deteriorating the response between 400 $\mathrm{Hz}$ and $500 \mathrm{~Hz}$. The peak response of the blade output only shows marginal reduction using this controller, as predicted by Fig. 13 .

The compensator transfer function is derived initially in the continuous time domain and then converted to a discrete time function using a first order hold approximation with a sampling 


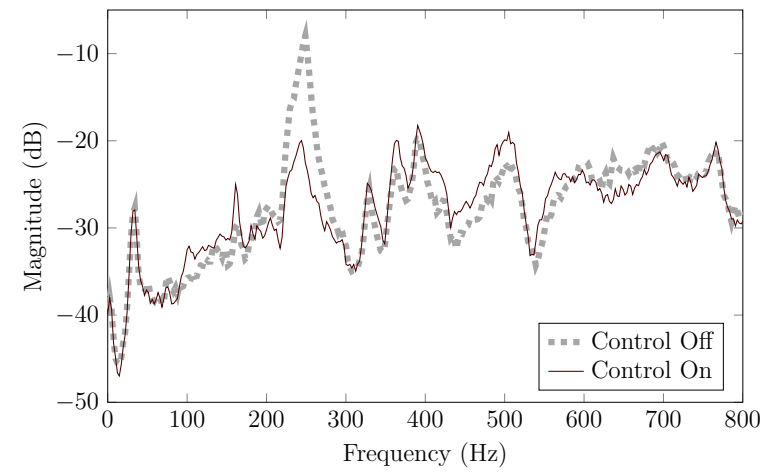

Fig. 15. Power spectral density of thrust block acceleration when disturbance shakers are excited by an uncorrelated random signal

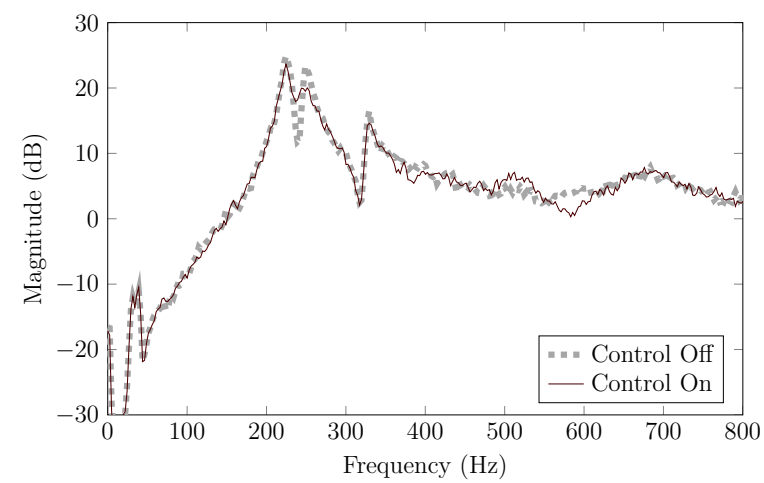

Fig. 16. Power spectral density of the summation of blade acceleration when disturbance shakers are excited by an uncorrelated random signal

frequency of $5 \mathrm{kHz}$. The resulting controller function is then implemented on the rig using a dSPACE rapid prototyping control system. The power spectral density of the acceleration response measured at the thrust bearing with and without feedback control when the blade is excited by a random white noise signal is shown in Fig. 15. This shows that the controller provides an attenuation of around $12 \mathrm{~dB}$ in the peak response relative to the uncontrolled case. The corresponding blade response for the same excitation is shown in Fig. 16 where it can be seen that although the peak response is only marginally reduced under the action of the controller it is not enhanced. As a result all of the controller design objectives have clearly been achieved therefore demonstrating the practical value of the proposed methodology.

\section{CONCLUSiON}

For many practical applications of active control it is not feasible to locate actuators and sensors in all regions where vibration attenuation is required. In a series of recent papers a geometric design methodology was introduced to provide a systematic procedure for optimising global performance with a single control loop. A drawback of this original method, however, was a requirement to assume that the local control path dynamics are minimum phase in order to guarantee both internal stability and that the controller is itself stable. In this paper the original methodology has been extended to provide a systematic procedure to obtain a stable and stabilising controller for the more general non-minimum phase case. A modified design freedom parameter has been introduced such that a controller can be implemented using the inverse of the minimum phase counterpart of the local control path transfer function. The theoretical results have been verified and the design procedure illustrated using a simulated beam vibration problem. A stable and stabilising controller was designed for this system to provide excellent reductions in global vibration levels relative to the uncontrolled case across a broad range of frequencies. Finally, the methodology has been validated experimentally using a rig that replicates the problems of vibration transmission in rotor blade systems. This specific problem provided the motivation for the development of the original geometric design methodology. The rig although having a near collocated actuator-sensor pair has non-minimum phase characteristics. It has been shown that despite this the new design methodology can be used to develop a strongly stabilising controller that provides significant attenuation of the transmitted vibration into the supporting structure without enhancing blade vibration.

\section{REFERENCES}

[1] Zhang, K and Scorletti, G and Ichchou, MN and Mieyeville, F, "Phase and gain control policies for robust active vibration control of flexible structures," Smart Materials and Structures, 22(7): 075025, 2013

[2] Kim, S-M and Oh, J-E, "A modal filter approach to non-collocated vibration control of structures," Journal of Sound and Vibration, 332(9): 2207-2221, 2013

[3] Derham, RC and Hagood, NW, "Rotor design using smart materials to actively twist blades," Proceedings of the American Helicopter Society 52nd Annual Forum. pp.1242-1252, Washington DC, 1996

[4] Riemenschneider, J and Opitz, S, "Measurement of twist deflection in active twist rotor," Aerospace Science and Technology, 16(3): 216-223, 2011

[5] Staple, A and Wells, D, "The development and testing of an active control of structural response system for the EH101 helicopter," Proceedings of the 16th European Rotorcraft Forum. 9, 1990

[6] Hartnell-Beavis, MC and Swinbanks, MA, "Some recent practical and theoretical developments in noise reduction in ships," Proceedings of the Conference on Vibration and Noise Levels in Ships at the Institute of Marine Engineers, 21: 28-38, 1976

[7] Daley, S and Wang, J, A geometric approach to the design of remotely located vibration control systems, Journal of Sound and Vibration. 318(4): 702-714, 2008

[8] Wang, J and Daley, S, "Broad band controller design for remote vibration using a geometric approach," Journal of Sound and Vibration. 329(19): 3888-3897, 2010

[9] MacMartin, DG and How, JP, "Implementation and prevention of unstable optimal compensators," American Control Conference. 2: 2190-2195, 1994

[10] Balini, H and Scherer, C and Witte, J, "Performance enhancement for AMB systems using unstable $\mathcal{H}_{\infty}$ controllers," Control Systems Technology, IEEE Transactions on. 19(6): 1479-1492, 2011

[11] Nyawako, DS and Reynolds, P, "Response-dependent velocity feedback control for mitigation of human-induced floor vibrations," Smart Materials and Structures. 18(7): 075002, 2009

[12] Rohlfing, J and Elliot, S and Gardonio, P, "Feedback compensator for control units with proof-mass electrodynamic actuators," Journal of Sound and Vibration. 331, 2012

[13] Fu, M, "Interpolation approach to $\mathcal{H}_{\infty}$ estimation and its interconnection to loop transfer recovery," Systems and control letters. 17(1): 29-36, 1991

[14] Doyle, JC and Glover, K and Khargonekar, P and Francis, BA, "Statespace solutions to standard $\mathcal{H}_{2}$ and $\mathcal{H}_{\infty}$ control problems," Automatic Control, IEEE Transactions on. 34(8): 831-847, 1989

[15] Levy, EC, "Complex-curve fitting," Automatic Control, IRE Transactions on. 1: $27-43,1959$ 